\title{
Application of Rapid Identification and Typing of Microorganisms in Detection of Food and Drug derived Microorganisms
}

\author{
Zhenguo Shen, Qian Sun* \\ Hainan College of Vocation and Technique, Haikou 570216, China \\ *Corresponding Author email: 370952@qq.com
}

Keywords: microbiological detection; PCR; MALDI-TOF mass spectrometry; ELISA; food and drug quality

\begin{abstract}
The detection of food and drug derived microorganisms is the main way to judge whether the quality of food and drug is qualified, and the application of the corresponding identification and typing technology can help to improve the detection efficiency. This paper briefly introduces several commonly used methods for rapid identification and typing of microorganisms, and analyzes the different characteristics of different technologies. Based on this, this paper focuses on the application of ELISA technology in food and drug derived microorganism detection, and the application value of the technology is confirmed by the detection effect.
\end{abstract}

\section{Introduction}

In recent years, the problem of high content of microorganism and poor quality of food and drug has occurred frequently, which has posed a serious threat to human health [1]. Therefore, the State Food and drug administration has paid much attention to the detection of food borne microorganism [2]. In order to ensure the rapid and accurate detection results, the key point is to apply the rapid microbial identification and typing technology, represented by ELISA technology, to the detection process [3-5]. Obviously, it is necessary to analyze the application methods of technology [6].

\section{Rapid Identification and Typing Technology of Microorganisms Commonly Used in the Detection of Food and Drug Derived Microorganisms}

\subsection{Fluorescence quantitative PCR Technology}

Fluorescence quantitative PCR technology is a kind of PCR technology. Fluorescent groups are added to the microbial reaction system [7]. By measuring the fluorescence signal in the reaction process, we can detect the content of microorganisms in food and medicine [8]. Fluorescent quantitative PCR technology was used to detect the content of microbes in food and medicine [9-10]. The results can be presented in a standard curve. Through the observation of the standard curve, the final test results can be obtained. However, it should be noted that, under the influence of sequencing technology, the fluorescence quantitative PCR technique is used to detect the content of food source microorganisms, and there may be some errors in the future.

\subsection{MALDI-TOF mass spectrometry}

MALDI-TOF mass spectrometry is a new technology of microbial detection. It can detect microbial content by decomposing the protein. Under the action of MALDI-TOF mass spectrometry, the charge of sample and matrix can be transferred to each other. At this time, with the help of corresponding software, we can get the relevant map of the results of microbial detection. By observing the map, we can identify the types of microorganisms in the samples and the content of different types of microbes. It is found that this method is effective when applied to the outbreak of foodborne diseases. In addition, it is also feasible to apply it to medicine and genetic engineering. 


\subsection{ELISA Technology}

ELISA technology, also known as enzyme linked immunosorbent technology. It is applied to the detection process of food source microorganism, and the detection speed is fast, and the accuracy of the detection results is high. At the time of detection, the antigen or antibody can be immobilized on the surface of the solid carrier. On this basis, enzyme is used to connect antibodies and antigens. Only when the reaction between enzyme and antibody and antigen is observed, can we identify the types of microbes in the samples and the contents of all kinds of microbes. Under the catalysis of enzymes, the sensitivity of ELISA technology to the detection of food derived microorganisms will be greatly improved. At present, the detection value of ELISA has been confirmed.

\section{Application of Rapid Microbial Identification and Typing Technology in Food Source Microbiological Detection}

This part takes ELISA technology as an example, expounds its application method in food borne microorganism detection, and observe the application effect of the technology.

\subsection{Instruments and reagents}

Centrifuges and micro sampler are the main instruments to detect microbial content by ELISA technology. Test reagents, including acetonitrile, methane and so on. Acetonitrile is generally analytically pure in the above two reagents, and it needs to be prepared. Acetonitrile water extraction method is as follows: fine sample $5 \mathrm{~g}$, placed in $50 \mathrm{ml}$ centrifuge tube. Add $20 \mathrm{~m}$ acetonitrile - water solvent and shake well. After $30 \mathrm{~s}$, at the speed of $5000 \mathrm{r} / \mathrm{min}, 10 \mathrm{mim}$ is centrifuged. After centrifugation, the supernatant of the sample solvent is obtained, and the final acetonitrile water extraction material can be obtained through distillation and water bath.

\subsection{Quantitative enzyme-linked immunosorbent assay}

Before the quantitative analysis of enzyme linked immunosorbent assay, a number of microporous strips should be prepared. Then the corresponding microorganism and 50 - $\mathrm{L}$ antibody were added to the microporous strip. When $2 \mathrm{H}$ was incubated at room temperature, the solution was distilled and the sample solution was obtained. In this study, different quantitative detection limits for different concentrations of reagents are shown in Table 1:

Table 1 quantitative detection limit

\begin{tabular}{cccc}
\hline $\begin{array}{c}\text { Reagent } \\
\text { concentration } \\
\left(\mathrm{ng} / \mathrm{L}^{-1}\right)\end{array}$ & $\begin{array}{c}\text { Standard deviation } \\
\left(\mathrm{ng} / \mathrm{L}^{-1}\right)\end{array}$ & $\begin{array}{c}\text { Detection limit } \\
\left(\mu \mathrm{g} / \mathrm{L}^{-1}\right)\end{array}$ & $\begin{array}{c}\text { Quantitative detection } \\
\text { limit }\left(\mu \mathrm{g} / \mathrm{L}^{-1}\right)\end{array}$ \\
\hline $18 / 21 / 32 / 35 / 38$ & 7.81 & 0.022 & 0.079 \\
\hline
\end{tabular}

In order to determine the microbial content of ELISA samples, whether there is variation in samples. In this study, the coefficient of variation (CV) measured by different samples was observed. The results are shown in Table 2:

Table 2 sample determination of variation coefficient

\begin{tabular}{cccc}
\hline Sample code & Sample A & Sample B & Sample C \\
\hline Coefficient of variation (\%) & 25.10 & 11.10 & 2.20 \\
\hline
\end{tabular}

\subsection{Results of microorganism detection}

In order to judge the microbial content of ELISA samples, the recovery rate of samples is reasonable. In this study, sample $A$, sample $B$ and sample $C$ were taken, and the recoveries of the three samples were calculated by corresponding software. The result of the calculation is shown in Table 3. 
Table 3 Sample recovery

\begin{tabular}{cccc}
\hline Sample code & Sample A & Sample B & Sample C \\
\hline Recovery rate (\%) & 98.5 & 97.8 & 123.0 \\
\hline
\end{tabular}

The above recovery results confirm the feasibility of the application of ELISA detection technology in the detection of microbial content. The difference between the results of A, sample B and sample $\mathrm{C}$ microbial content determination and the actual results was further observed. The results are shown in Table 4:

Table 4 Sample microbe test results and actual situation

\begin{tabular}{cccc}
\hline Sample code & Sample A & Sample B & Sample C \\
\hline detection result $(\mu \mathrm{g} / \mathrm{kg})$ & 5.41 & 6.18 & 2.21 \\
Physical truth $(\mu \mathrm{g} / \mathrm{kg})$ & 5.43 & 6.20 & 2.23 \\
\hline
\end{tabular}

Observation table 4 can be found that there is no significant difference between the results of microbial detection of samples and their actual results. The above results show that the application of ELISA technology to microbial detection process can effectively improve the accuracy of detection results.

\section{Conclusion}

To sum up, PCR, MALDI-TOF mass spectrometry and ELISA technology have their own advantages. This topic provides a feasible reference for the rapid identification and typing technology of microorganisms. Therefore, relevant departments in China should actively apply relevant technologies to the detection process. On the basis of strictly controlling the factors such as temperature and time, the detection efficiency is improved and the accuracy of detection results is improved.

\section{References}

[1] Stöckel S, Kirchhoff J, Neugebauer U, et al. The application of Raman spectroscopy for the detection and identification of microorganisms[J]. Journal of Raman Spectroscopy, 2016, 47(1):89-109.

[2] Garza-García L D, Pérez-González V H, Pérez-Sánchez O A, et al. Electrokinetic Mobilities Characterization and Rapid Detection of Microorganisms in Glass Microchannels[J]. Chemical Engineering \& Technology, 2015, 34(3):371-378.

[3] Karlsson R, Gonzales-Siles L, Boulund F, et al. Proteotyping: Proteomic characterization, classification and identification of microorganisms--A prospectus[J]. Systematic \& Applied Microbiology, 2015, 38(4):246-257.

[4] Curtoni A, Cipriani R, Marra E S, et al. Rapid Identification of Microorganisms from Positive Blood Culture by MALDI-TOF MS After Short-Term Incubation on Solid Medium[J]. Current Microbiology, 2016, 74(1):1-6.

[5] Nakanishi T. [Rapid Identification of Infectious Microorganisms in Clinical Samples by MALDI-TOF MS Analysis] [J]. Rinsho Byori the Japanese Journal of Clinical Pathology, 2015, 63(4):465-71.

[6] Dolci P, Alessandria V, Rantsiou K, et al. 7 -Advanced methods for the identification, enumeration, and characterization of microorganisms in fermented foods[J]. Advances in Fermented Foods \& Beverages, 2015, 12(1):157-176.

[7] A. Fröhling, M. Erhard, P. Muranyi, et al. Rapid identification of microorganisms by MALDI-TOF MS[J]. Landtechnik, 2015, 70(2):25-30. 
[8] Wang M C, Lin W H, Yan J J, et al. Early identification of microorganisms in blood culture prior to the detection of a positive signal in the BACTEC FX system using matrix-assisted laser desorption/ionization-time of flight mass spectrometry[J]. J Microbiol Immunol Infect, 2015, 48(4):419-424.

[9] Niimi H, Ueno T, Hayashi S, et al. Melting Temperature Mapping Method: A Novel Method for Rapid Identification of Unknown Pathogenic Microorganisms within Three Hours of Sample Collection[J]. Scientific Reports, 2015, 5(12543):1-13.

[10] Reen F J, Romano S, Dobson A D W, et al. The Sound of Silence: Activating Silent Biosynthetic Gene Clusters in Marine Microorganisms[J]. Marine Drugs, 2015, 13(8):4754-4783. 